\title{
Analysis of BP Neural Network Applied in Enterprise Financial Warning
}

\author{
Hang Zeng \\ Xijing University, Xi'an Shan'xi, 710123,China
}

\begin{abstract}
Keywords: BP neural network, Enterprise financial crisis warning, Warning signal.
\end{abstract}
\begin{abstract}
In the 21st century, high-tech society, the information age, more and more on the "Network" emerging term, as we respect these new terms confusing and difficult to understand, but also had to lament these new terms of social development made contribution. And on the "Network" word, also has its own value and significance of the epoch. Factors Chinese enterprises financial distress has its inevitability and unique, and therefore, according to the researchers supply situation and related indicators of the current financial accounting information in China, plus the disclosure of accounting information quality indicators, to build a number of early warning indicators system and a variety of early warning system, combined with the relatively fast development of the city, to test analyzed the financial data, to jointly promote and improve the accuracy of the financial crisis early warning and the like. This article is mainly on BP neural network and its application in the enterprise financial crisis of the analysis.
\end{abstract}

\section{Introduction}

In the enterprise on the financial crisis early warning, has a very important value analysis, on this issue, many scholars in related industries are also constantly research and exploration, and also have very impressive results. Although China for the start of the study and analysis of enterprise financial crisis of late, but the results achieved is very substantial, for the financial crisis warning also it has a very prominent significant contribution. Our country has come to a stage of rapid development, finance is the lifeblood of the entire enterprise, but also understand their enterprise as an important reference. However, with the continuous development of state and society, the lifeblood of enterprises also suffer to a certain extent a serious crisis, the crisis on the verge of the lifeblood of enterprises, how to strengthen preventive measures, our country is also doing research related aspects. Eventually, a new term "BP" neural network began to be widespread attention and application, but also the whole enterprise financial crisis has made huge success and contribution.

\section{Related contents about financial crisis warning}

The financial crisis is of great value. Financial crisis refers to the financial management of enterprises; enterprises are facing greater danger and opportunity. Enterprise financial operation is a fundamental of business development, the financial crisis threatening the survival and development of enterprises. Current social development, many uncertain factors affecting financial operations and management, this situation, enterprises must deal with the crisis and master crisis measures in order to enhance the company's ability, fighting with all the uncertainties, is not surprised, ensure a stable, reliable and sustainable development. Financial management of modern enterprises and people's economic lives, financial management and operational level, as well as the quality of the financial information not only related to the survival of enterprises, but also about the stability of the overall socio-economic development. Examine the possibility of implementing the necessary solution to the financial crisis, is also quite necessary. Financial crisis is the need to urgently address the problem, relevant 
departments of the State as the solution to the financial crisis, developing a financial model and implementation plan. Sound financial management system of enterprises, is also dealing with crisis very favorable way. An important topic for modern enterprises, about the handling and management of financial crises, taking scientific high-tech-related measures, applied in enterprises in financial distress. On accounting information supply in China based on established categories relating to the early warning system, the early-warning index system include ability to service its debt, and asset management, as well as the development of the disclosure quality of accounting information and cash flows, such as the six. BP neural network and application in enterprise financial crisis quite extensive and excellent, especially after standard of inspection, the discriminant accuracy of the model has been significantly improved, and has considerable advantages. In the financial crisis, and develop appropriate early warning model is also more critical components. Once the financial crisis broke out in the enterprise, across an enterprise in financial trouble, and some may even go bankrupt. In the financial management of enterprises, and financial crisis is one of the biggest traps, on early-warning of enterprise financial crisis, financial crisis prevention, such as content, financial management of enterprises has also become a top priority; financial management at the enterprises financial distress is also one of the most powerful tool.

\section{Study and analysis of BP neural network in financial crisis warning mode}

Development of financial early warning model has its patterns and long process, developed by its gradual evolution from a single variable to multivariable statistical methods to the non-statistical, on the pattern from single to mixing, such an overall development process. On early warning models, typically there are several, but the practicality of these methods ultimately due to more stringent warning indicators and a certain degree of interference and restrictions. Neural network model with artificial processing unit connected to form a network, but also has the characteristics associated distributed storage, and ultimately achieve the structure and function of the human brain simulation. Neural network model in dealing with the needs and consider many factors imprecise conditions, etc., play an essential role, but also the financial crisis early warning made a very important contribution and value.

BP neural network in accordance with the national financial crisis early warning, and come up with the financial crisis prediction model is a multi-layer network structure, including input, output and three hidden layer structure. About the input layer of the model, the number of neurons contained herein is determined by the number of financial indicators have been determined. And the input of the model is corporate financial indicators. This study speaks to critical indicators modeling than the direct use of financial indicators modeling better predict a more significant effect, while building two related models, so that the number of neurons in the input layer corresponds also been two cases . No matter what kind of modeling, normalization must go through a process input, to remove dimensional impact, but also avoid the weight or the input is too large, resulting in a network into a relatively saturated state. On hidden layers to determine the relevant literature proved that, under certain specific conditions, BP neural network multilayer can come close to the mapping relationship by any accuracy. The same comparison with a hidden layer, two hidden layers for accurate forecasting to improve its ability to not be able to have a good play, so the use of three-layer neural network, but also help improve its forecasting capabilities. About the number of neurons in the output layer depends mainly on its output category. Neurons are what we call the output layer of the network, and training in its sample, there is also a corresponding conversion amount. About BP neural network applications in the enterprise financial crisis, countries are vigorously promoting and doing research, neural network model mentioned in this article, but also fully demonstrated in early warning, neural network technology has strengths and capabilities, it also has a strong two properties, one is operational, the other is its normative, two properties so that the entire neural network to better play its role better applications in enterprise financial crisis. 


\section{About how to choose sample and index}

The first is about the choice of the sample, according to the study years of sample data so that more advanced model predictive effect, through the comparison of the two major listed companies, the final decision on the implementation of the company special treatment. Many scholars in practice, according to a year before the advent of early warning indicators, although this can have a very high accuracy, but it is not the most realistic forecast, so research should try to select the four years before the index data do twenty-three sample. Necessary financial indicators also need to select the previous five years or more sample data as samples. Nor does it at the same time in a given year to the election as a cross-sectional sample, select the sample, we also need to select the appropriate number. The second is about the choice of financial indicators, the neural network model has a natural characteristic, or a non-linear model, nonlinear relationship with respect to the data, it can be very accurately identify and simulate it, you do not need to assume more variables probability and normal.

\section{Analysis of application and result of neural network model}

Application of neural network model, which are modeled according to two different ways to determine the key warning indicators, primarily through direct modeling and screening of two tests, plus the way the neural network model and construct a complete empirical tests.

First mentioned by way of direct modeling complete test results, three feed forward neural network is achieved by a research network applications, network model of direct modeling use of early warning indicators reached, before training the neural network during its creation, by randomly selected sample data is divided into two blocks, one is used to train the neural network, the other is used to simulate neural networks. Network training process, usually is relatively quick way is to use a training function, by writing language program, continue to experiment, using several parameters related to different training, based on the use of two or three years ago and the first four years of relevant financial data, and normal sample data for the corresponding year, specific training corresponding neural network, preparation of training and simulation program. By the second year before the relevant financial data made training network, has been associated neural network effectively warning.

Second, it is the result of the neural network model testing and screening applications. Through the 50 indicators tested, key warning indicators to filter, build a new neural network model. According to the relevant results that, with ST nearer, it's different from normal will be more and more appear on the index, which is the closest to reality check. Through research and comparison, the final decision is to select a certain amount of indicators about the model as input variables. And this testing process with the inspection process is similar to direct modeling are probably based on a sample of pre-crisis twenty-three four years, as well as normal samples tested neural network model of the corresponding year. Corresponding determination result analysis, prediction accuracy of the model show, ST's relatively high ratio of non-ST companies accuracy needed. The model includes all of the results are better than accuracy not previously tested to be higher.

\section{Conclusions}

In the financial sector, such as the body's central nervous system, it can predict everything positive and negative whole corporate earnings loss, etc., is also inseparable from the development process of the enterprise financial efforts and cooperation in ensuring better business profitability and development, we must also eliminate all possible financial crisis, so that the financial work and orderly operation. BP neural network is also looking forward to play its real value in our enterprise financial crisis, in order to better the development of enterprises have made outstanding contributions. About enterprise financial crisis now processing method described in this paper, leaving we learned about a new "network" term, BP neural network, high-tech society, we expect more of the same high-tech terms appear, We hope that these new high-tech terms, for the social and economic 
development can make a more significant contribution. Our country and society, has been developed to the point where we cannot imagine, this time destined to be dominated by high-tech product, but also because we are a number of high-tech society, but proud and excited.

\section{References}

[1] Yao Yibo, Wang Jiliang. Study on improving BP network practice speed, Information technology. 2010(01)

[2] Zhou Min, Wang Xinyu. Enterprise financial crisis and Fuzzy Optimum Neural Network, Journal of Management, 2010(03)

[3] Zhang Qiushui, Luo Linkai, Liu Jinming. Chinese Listed Companies' Financial Distress Prediction Based on SVM,Computer application, 2011(S1)

[4] Chen Jing. An Empirical Analysis of Listed Companies' Financial deterioration prediction, Accounting Research, 2011(04)

[5] He Changzheng, Li Xiaofeng, Yu Hai. Improvement and application of new BP Artificial Neural Network Model,Practice and understanding of Mathematics 2010(04)

[6] Zhang Zaixu, Song Jiekun, Zhang Yu. An enterprise financial crisis warning new model based on based on support vector machine, Journal of China University Of Petroleum (natural science edition), 2010(04)

[7] Qiu Yulian, Zhu Qin. Financial warning method based on SVM, Statistics and Decision,2011(16)

[8] Yang Baoan, Ji Hai, Xu Jing, Wen Jinxiang. Application of BP neural network in enterprise financial crisis warning, Predication,2011(02)

[9] Yao Yibo, Wang Jiliang. Study on improving BP network practice speed,Information Technology,2011(5)

[10]Zhang Zaixu, Song Jiekun, Zhang Yu. Enterprise financial crisis based on support vector machine new warning, Business management,2010(4) 